\title{
Corela
}

Cognition, représentation, langage

HS-6 | 2007

Cognition, discours, contextes

\section{Discours, mémoires et contextes : à propos du fonctionnement de l'allusion dans la presse}

\section{Sophie Moirand}

\section{(2) OpenEdition}

\section{Journals}

Édition électronique

URL : http://journals.openedition.org/corela/1567

DOI : $10.4000 /$ corela. 1567

ISSN : 1638-573X

Éditeur

Cercle linguistique du Centre et de I'Ouest - CerLICO

Référence électronique

Sophie Moirand, « Discours, mémoires et contextes : à propos du fonctionnement de l'allusion dans la presse », Corela [En ligne], HS-6 | 2007, mis en ligne le 01 novembre 2007, consulté le 10 décembre 2020. URL : http://journals.openedition.org/corela/1567 ; DOI : https://doi.org/10.4000/corela.1567

Ce document a été généré automatiquement le 10 décembre 2020.

\section{(c) (i) (2) (2)}

Corela - cognition, représentation, langage est mis à disposition selon les termes de la licence Creative Commons Attribution - Pas d'Utilisation Commerciale - Partage dans les Mêmes Conditions 4.0 International. 


\title{
Discours, mémoires et contextes : à propos du fonctionnement de l'allusion dans la presse
}

\author{
Sophie Moirand
}

1 L'analyse du discours française (ADF), essentiellement centrée à l'origine sur les discours politiques, s'est construite dans les années 1970-1980 en dehors du paradigme cognitiviste (voir 1.1. infra). Ce qui explique peut-être l'absence de référence aux activités cognitives des locuteurs/interlocuteurs dans un certain nombre de travaux en France, comme celle de l'entrée "cognition» dans le Dictionnaire d'analyse du discours paru en 2002 (Charaudeau et Maingueneau éds) ${ }^{1}$. Ce qui n'est pas le cas d'autres courants d'analyse, plus proches de la pragmatique anglo-saxonne, qui ont pris rapidement un tour cognitif, avec H.P. Grice d'abord, puis D. Sperber et D. Wilson, ainsi que A. Reboul et J. Moeschler dans le champ francophone. Ce qui n'est pas non plus le cas du courant de la Critical Discourse Analysis, représenté par T.A. van Dijk, dont les objets d'étude rejoignent ceux de l'ADF, par exemple les discours politiques.

2 T. van Dijk, en se démarquant des courants sociolinguistiques et interactionnistes qui posent une relation directe entre les énoncés et les contextes situationnels (ou situations), postule en effet, dans la reconstruction du contexte par les participants de la communication à partir des éléments dits pertinents de la situation, une «interface cognitive ", c'est-à-dire la médiation du cognitif entre la situation de communication (d'ordre social) et les structures de surface du discours (ce que l'on peut assimiler à la «matérialité discursive» de la tradition française: les formes de la langue). Il n'est d'ailleurs pas étonnant que van Dijk, qui s'est intéressé dans les années 1975-1980 à la modélisation de la compréhension et de la production des textes avec W. Kintsch, propose aujourd'hui un «modèle de contexte » qui repose sur les représentations sociocognitives que les participants se font de la situation et non pas sur la situation « concrète », telle que pourrait l'appréhender un observateur extérieur.

3 Je ne commenterai pas le modèle de van Dijk ${ }^{2}$ dont je partage la conception de l'interface cognitive. Je voudrais plutôt montrer ici que si les mots cognition ou cognitif semblent 
absents des textes principaux de l'analyse du discours française originelle, ils me paraissent cependant avoir été inscrits "en creux ", dès qu'il s'est agi de théoriser le fonctionnement du discours, en particulier derrière les mots «mémoire " et «oubli » (mais pas seulement) et dans leurs liaisons implicites avec les notions de contexte (historique, social ou culturel) et celles de pensée et d'expérience humaine :

- qu'il s'agisse de postuler le rôle de la mémoire cognitive (à court et à long terme), dans le cadre d'une linguistique textuelle qui s'intéresse aux phénomènes de coréférence ou dans le cadre d'une logique naturelle qui étudie la transformation des objets de discours au fil du texte (dans l'intratexte) ;

- qu'il s'agisse de la mémoire de l'histoire fortement présente dans l'analyse du discours française et dans la sémantique discursive théorisée par M. Pêcheux, à savoir que si le sujet n'est pas la source du sens, c'est que « le sens se construit dans l'histoire à travers le travail de la mémoire, l'incessante reprise du déjà-dit » (Maldidier 1990);

- qu'on pense en termes de dialogisme, avec M. Bakhtine, notion omniprésente actuellement en $\mathrm{AD}$ mais déjà présente dans les travaux de J. Authier-Revuz (1982 par exemple), celui du mot « qui n'oublie jamais son trajet » et donc les discours qu'il a déjà rencontrés (Moirand 2004c, 2005), ou en termes de pré-construit, d'interdiscours et de la théorie des deux oublis (Pêcheux), et donc en termes de discours transverses et de mémoire interdiscursive (Courtine et Lecomte, voir infra) ;

- qu'on pense enfin en termes de " mémoire collective » vs « mémoire individuelle » (Halbwachs), et en relation (ou non) avec les travaux actuels en psycholinguistique, en sciences cognitives ou en biologie (Petit 2006 pour une synthèse récente), les mémoires épisodique, perceptive, procédurale, sémantique et la mémoire de travail (les cinq mémoires de Tulving 1995, par exemple).

On essaiera ici de démêler d'abord ce qu'on entend par mémoire en analyse du discours, depuis Courtine et Lecomte, et dans le cadre actuel d'une sémantique discursive renouvelée par la prise en compte des relations entre le sujet (social) et la réalité (sociale) telle qu'il la perçoit, qu'il l'interprète et qu'il la catégorise avant de la représenter pour d'autres.

On s'interrogera ensuite, à la lumière des analyses effectuées sur des corpus de la presse quotidienne française, sur ce que les mots et les dires transportent avec eux (donc sur les fils verticaux du discours) lorsqu'ils s'inscrivent dans la matérialité discursive (donc dans le fil horizontal du discours). Ce qui permettra de discuter des formes de mémoire qui sont alors, me semble-t-il, convoquées et du rôle de l'allusion dans la mémorisation et le rappel, voire la construction, des savoirs.

On montrera enfin comment l'appel à la mémoire des mots et des dires intervient dans l'éclairage proposé par l'énonciateur au fil du déroulement du texte (l'intratexte), en particulier dans des configurations discursives telles que l'explication médiatique ou l'argumentation, c'est-à-dire dans le cadre de la schématisation élaborée par J.-B. Grize : une organisation raisonnée de contenus de pensée visant à modifier les représentations cognitives des destinataires. 


\section{Mémoire et discours : les lieux d'une rencontre}

7 La mémoire est une notion quasiment absente des dictionnaires de linguistique et de sciences du langage du début des années 1970 en France, de même que des index des ouvrages qui sont parus en grand nombre dans les décennies 1960/1970 en linguistique. Signalée en entrée dans Le Langage (Pottier éd. 1973), elle l'est sans qu'aucun lien explicite soit fait avec la langue, le langage ou le discours. C'est à travers la diffusion en France de ce qu'on a appelé un temps «la grammaire de textes» qu'elle finit par rencontrer justement «le texte» dans les années 1975-1980, et parce qu'elle se trouve alors au croisement de travaux de psycholinguistes et de linguistes du texte : l'ouvrage Il était une fois ... Compréhension et souvenirs de récits (Denhière éd. 1984) constitue un exemple de cette première rencontre.

8 Mais dans la tradition de l'analyse du discours française, elle me semble d'abord apparaître "en creux», lorsque M. Pêcheux propose une conception du sujet liée à sa "théorie des deux oublis ", que je rappellerai brièvement ici (c'est moi qui souligne en italiques) :

Sous le terme d'« oubli », que Michel Pêcheux arrache à son acception psychologique, tente de se penser l'illusion constitutive de l'effet sujet, c'est-à-dire l'illusion pour le sujet d'être la source du sens. Dans l'« oubli n ${ }^{\circ} 1$ », le sujet « oublie », autrement dit refoule, que le sens se forme dans un processus qui lui est extérieur [...]. L'« oubli n ${ }^{\circ}$ 2 » désigne la zone où le sujet énonciateur se meut, où il constitue son énoncé, posant des frontières entre le « dit » et le rejeté, le «non-dit ». (Maldidier 1990: 34-35)

La théorie des deux oublis est alors reliée à la psychanalyse plutôt qu'à la mémoire : à l'insconcient pour l'oubli $n^{\circ} 1$ (par définition inaccessible au sujet), au préconscient ${ }^{3}$ pour l'oubli $n^{\circ} 2$, qui renvoie aux mécanismes énonciatifs repérables dans la matérialité discursive. Ce qui fixe du même coup un objectif à l'analyse du discours : celui de l'oubli n ${ }^{\circ} 1$, c'est-à-dire la saisie de l'interdiscours, comme le rappelle Denise Maldidier (ibidem). La théorie des deux oublis, présentée dans Pêcheux 1975 sera, comme le rappelle D. Maldidier (ibidem), « vite critiquée, puis abandonnée », non sans avoir ouvert la voie à la notion de mémoire discursive...

\subsection{La mémoire discursive dans l'ADF}

Proposée au début des années 1980 par le Groupe de Recherche en Analyse du Discours (université Grenoble 2), c'est dans la thèse de J.-J. Courtine sur le discours communiste adressé aux chrétiens que la notion de mémoire discursive paraît la plus élaborée (dans Langages 62, 1981). Notion qui surgit d'une rencontre entre la philosophie (Foucault), l'histoire (Braudel), l'idéologie (Althusser) et la théorie du discours (Pêcheux), elle se défend de tout ancrage cognitif, comme tiennent à le rappeler ses deux principaux promoteurs, J.-J. Courtine et A. Lecomte :

Ce que nous entendons par le terme de «mémoire discursive " est distinct de toute mémorisation psychologique du type de celle dont les psycholinguistes s'attachent à produire la mesure chronométrique (ainsi pour prendre un exemple récent, le travail de Kintsch et van Dijk (75) sur les processus cognitifs impliqués dans la mémoire des textes). La notion de mémoire discursive concerne l'existence historique de l'énoncé au sein de pratiques discursives réglées par des appareils idéologiques [... ] (Courtine 1981 : 52-53) 
[...] des recherches contemporaines (Foucault, de Certeau) ont mis l'accent sur l'hétérogène, sur l'existence parfois contradictoire de l'objet discursif (Courtine), sur les phénomènes d'incise, de discours transverse (Pêcheux), d'interdiscours [...] axe vertical où viennent interférer des discours déjà tenus, des discours antagonistes ou des discours voisins, axe enfin où on s'autorise à localiser une mémoire, en entendant par là non la faculté psychologique d'un sujet parlant, mais ce qui se trouve et demeure en dehors des sujets, dans les mots qu'ils emploient [...] (Lecomte 1981 : 71)

Il s'agissait alors de fonder l'analyse du discours sur une théorie essentiellement discursive en tenant compte d'une part de la langue, d'autre part de l'histoire qu'on appréhende "à partir de la contradiction des forces matérielles qui s'y affrontent " (Courtine 1981 : 121). La notion de mémoire discursive permet ainsi de rendre compte du fait que toute production langagière fait circuler des formulations antérieures, du déjàdit, du déjà-énoncé... Elle constitue alors une sorte de reformulation de l'interdiscours de Pêcheux.

12 Mais, près de dix ans plus tard, dans la présentation de sa thèse d'État, Courtine esquisse un bilan mitigé des travaux qui se sont développés en analyse du discours : recentrage de l'analyse sur le fil du discours (plutôt que sur l'interdiscours), sur l'horizontalité d'une séquence discursive souvent énoncée par un seul sujet, descriptions effectuées d'un point de vue formel et grammatical ou bien interactif et conversationnel qui abandonnent l'articulation du texte avec les conditions historiques de sa production, remplacement d' une analyse $d u$ discours qui voulait articuler histoire et linguistique par des analyses de discours qui ont pour la plupart abandonné un tel projet (Courtine 1989 : 12-14).

C'est cependant la notion de mémoire discursive élaborée par Courtine que j'ai reprise et que j'ai retravaillée récemment sur des corpus de presse constitués autour d'événements scientifiques ou technologiques à caractère politique (voir Moirand 2007a, chap. 4 pour une synthèse).

\subsection{La mémoire discursive « revisitée »}

Confrontée à la circulation des discours telle qu'on peut la rencontrer dans la représentation des événements, c'est-à-dire aux différentes formes de paroles citées, empruntées, représentées, reformulées, évoquées, imaginées, j’ai décidé en effet d'aborder les discours non pas à travers la notion de situation, non pas avec les formes syntaxico-sémantiques du discours rapporté, mais à travers un cadre dialogique de l'énonciation tel que l'a proposé Bakhtine, dans ses liens avec la pensée, la culture et l'expérience humaine, tel que je l'ai compris et interprété à travers la diversité des textes traduits en français de Bakhtine/Volochinov, et tel que je l'ai articulé aux notions d'interdiscours et de mémoire discursive de l'analyse du discours française :

[...] un énoncé ne peut pas ne pas être, également, à un certain degré, une réponse à ce qui aura déjà été dit sur l'objet donné, le problème posé, quand bien même ce caractère de réponse n'apparaîtrait pas distinctement dans l'expression extérieure. La réponse transpercera dans les harmoniques du sens, de l'expression, du style, dans les nuances les plus infimes de la composition [...]. Car notre pensée elle-même - que ce soit dans les domaines de la philosophie, des sciences, des arts - nait et se forme en interaction et en lutte avec la pensée d'autrui, ce qui ne peut pas ne pas trouver son reflet dans les formes d'expression verbale de notre pensée. (Bakhtine $1984: 300)$ 
Il m'a paru en effet que si Bakhtine ne parle pas de cognition ni même de mémoire, ces notions sont inscrites "en creux» dans sa conception des harmoniques dialogiques comme dans celle de l'autodialogisme. Il m'a paru d'autre part que ce qui faisait la spécificité des discours de la presse sur lesquels je travaillais, ce n'était pas l'inscription des relations interpersonnelles entres les acteurs producteurs d'énonciations singulières, mais les relations interdiscursives entre les discours qui y étaient représentées, et qui fonctionnent «en écho » à l'intérieur d'un article, d'un numéro, d'un même titre de presse, et plus globalement d'un média à un autre.

16 Ainsi, au fil des événements analysés, on a rencontré, inscrits dans la matérialité discursive, des mots et des formulations qui jouent le rôle de déclencheurs mémoriels sans qu'on puisse réellement parler de " discours rapporté », ou qui semblent renvoyer à des représentations relevant de domaines de mémoire à court terme ou à long terme :

1. Alerte au soja fou

Bruxelles n'a pas tiré les leçons de la vache folle

(Libération)

2. Le drame serait que les oiseaux migrateurs volent vers l'Afrique qui ne dispose

d'aucun réseau sanitaire pour contenir le Tchernobyl aviaire

(Paris-Match)

3. L'OGM ou la faim?

(Libération)

4. « Je ne suis pas un faucheur volontaire»

(Corinne Lepage, France-Inter, 16/01/2007)

17 Ainsi non seulement les dires sont porteurs de mémoire (reprise du mot de l'autre dans l'ex.4), mais certaines constructions inscrivent des discours antagonistes (ex.3), et certains mots eux-mêmes (la vache folle, Tchernobyl) ou même certains sèmes ('maladie', 'contamination'... inscrits dans fou/folle, ) transportent avec eux la mémoire des événements qu'ils désignent.

Ce que j'ai appelé alors « la mémoire des mots », celle inscrite par exemple dans des motsévénements (tels Tchernobyl, Bhopal, la vache folle... - Moirand 2007a : 56-58) m'a paru rejoindre certains conceptions de la praxématique, pour laquelle «les unités lexicales sont bel et bien des praxèmes » [...], « c'est-à-dire des outils d'analyse du réel en relation avec des pratiques socioculturelles" (Nyckees : 258-260), et en particulier ce que Paul Siblot développe dans sa conception du dialogisme de la nomination : que « la nomination est un acte de catégorisation, une praxis qui est simultanément sociale et linguistique " (Detrie, Siblot, Vérine éds 2001 : 205-206), et que «nommer ce n'est pas seulement se situer à l'égard de l'objet, c'est aussi prendre position à l'égard d'autres dénominations du même objet, à travers lesquelles des locuteurs prennent également position " (Siblot 1997 : 55). Or les catégorisations opérées lors des actes de nomination des événements ou des acteurs des événements relatés dans les médias relèvent bien d'opérations cognitivolangagières qui reposent à la fois sur des expériences et des connaissances ainsi que sur 
les discours qui les organisent et les formulent, donc sur une mémoire cognitive (mémoire sémantique? mémoire épisodique ? Cela sera discuté en 2. infra).

Prendre la parole, qu'il s'agisse d'un journaliste ou d'un acteur convoqué par les médias, pour relater un événement, cela consiste en effet :

- à évaluer (Volochinov 1981 : 190) le contexte situationnel au sens large, et il s'agit donc bien d'une représentation mentale que l'on reconstruit du contexte (au sens de van Dijk supra), et donc de l'événement;

- à effectuer des actes de catégorisation à travers des opérations de nomination, de désignation et de caractérisation de l'événement ainsi que des acteurs de cet événement, ce qui suppose de faire appel aux mots et aux constructions de la langue utilisée, tels qu'on les a emmagasinés en mémoire, c'est-à-dire avec les sens qu'ils ont pris dans les situations où on les a rencontrés auparavant, y compris ceux dont on est pas toujours conscients ;

- à effectuer ces actes en direction de classes de destinataires dont on a également une représentation et en fonction de la représentation que l'on veut donner de soi : on retrouve la schématisation conceptualisée par J.-B. Grize dans sa théorie argumentative, dont on reparlera en 3. infra.

Lors des événements désignés aujourd'hui par «la crise des banlieues » (automne 2005) ou « la crise du CPE » (hiver-primtemps 2006), on a pu relever dans la presse quotidienne un certain nombre d'actes de nominations qui ont contribué à leur construction (Moirand 2006b). Il y avait par exemple ceux qui désignaient les faits eux-mêmes (tensions, troubles, incidents, affrontements, heurts, échauffourées, harcèlements, déchainements, débordements, explosion/éruption/flambée de violence, émeutes, guérilla, violences urbaines $\rightarrow$ crise pour le premier ; contestation, mobilisation, mouvement, heurts, incidents, échauffourées, affrontements, violences, violences policières, conflit $\rightarrow>$ crise pour le second). Il y avait également ceux qui désignaient les acteurs de ces événements, des jeunes dans les deux cas, mais qui ont été peu à peu re-nommés au fil du déroulement de l'événement selon des paradigmes divergents. Dans le premier cas, il s'agissait de jeunes, des jeunes des cités; des adolescents, des gamins, des enfants, des mômes; de petits voyous, de jeunes casseurs, des jeunes émeutiers; des voyous, des émeutiers, des incendiaires, des casseurs encagoulés, des "encagoulés", des insurgés, des groupe d'émeutiers, "un ennemi très mobile », la racaille... Dans le deuxième cas, il s'agissait d'étudiants, de lycéens (avec leurs parents, avec les salariés du public et du privé); d'étudiants en lutte/en grève/en colère; d'anti-CPE, de manifestants, de grévistes, de bloqueurs, de porteurs de banderoles; de hordes... d'étudiants radicaux, de sauvages, de casseurs, de voyous; d'anarchistes, de trotskystes, de radicaux trotskystes, d'anarcho-syndicalistes, etc.

21 On peut imaginer ici les phénomènes de catégorisation telles qu'ils s'élaborent dans la tête des locuteurs invités à prendre la parole: les représentations mentales et les conceptualisations qui se construisent à travers les expériences que l'on a du monde ainsi qu'à travers les discours qui les organisent et les configurent. Mais on peut également imaginer comment ces formulations influent sur la construction de l'événement luimême et la perception qu'en ont les destinataires des discours diffusés par les médias. Si analyser les constructions discursives de l'événement repose en partie sur le repérage des inscriptions de ces opérations dans la matérialité textuelle (ce qui est l'hypothèse 
d'une approche linguistique du discours), cela consiste également à traquer les traces d'une interdiscursivité (les fils verticaux du discours, les discours transverses...) qui s'inscrit dans les mots, les constructions, les dires repérables dans l'ordre du discours (le fil horizontal, l'intratexte...). Ainsi les catégorisations opérées par les locuteurs lorsqu'ils prennent la parole inscrivent en elles-mêmes une fois formulées une histoire et donc des domaines de mémoire qui relèvent de l'interdiscursif, tout en contribuant d'autre part à la construction des mémoires collectives. Ce sont en tout cas les hypothèses actuelles que je formule et que je voudrais soumettre à discussion.

\section{Du rôle de l'allusion dans la mémorisation et le rappel des savoirs}

Dans les travaux que j'ai conduits ces dernières années sur le traitement par les médias des événements scientifiques ou technologiques à caractère politique (l'affaire du sang contaminé, la crise de la vache folle, le poulet à la dioxine, la grippe aviaire, les controverses autour des OGM), j'ai fait travailler la notion de dialogisme en l'articulant à celles d'intertexte et d'interdiscours (Moirand 2007b). À côté des formes «situées » de paroles et d'énoncés rapportés, qui donnent à certains genres de l'information une texture énonciative particulière (une hétérogénéité montrée qui se manifeste par une construction plurilogale de l'intertexte), je me suis attachée aux formes de ce que j'ai appelé l'hétérogénéité suggérée, caractéristique des textes à énonciation subjectivisée (les éditoriaux, les commentaires, les points de vue, les dessins de presse...). Ces genres de texte ont contraint en effet à déplacer le repérage des formes de citation et de discours rapporté vers celui des formes de l'allusion: sont alors apparus des mots, des formules, des figements et des défigements, des constructions syntaxiques, qui fonctionnent comme autant de rappels mémoriels de dires, de faits et d'événements antérieurs.

J'ai donc formulé l'hypothèse qu'il existe une mémoire interdiscursive propre aux médias, et que cette mémoire contribue à donner un « air de famille » à une série d'événements (la contamination du sang, la transmission du prion à l'homme, la transformation génétique des plantes...) qui, pour des scientifiques, n'ont rien en commun. En élargissant depuis les corpus à d'autres familles d'événements, il m'est apparu que les différentes formes d'allusion n'étaient peut-être pas de nature strictement discursive, et qu'on ne pouvait pas, comme l'avaient fait Courtine et Lecomte dans une perspective de construction théorique disciplinaire, exclure les bases cognitives de la mémoire dans la construction médiatique des relations entre discours et histoire. Cela mérite en tout cas un débat.

\subsection{Des lieux d'inscription de la mémoire}

Au fil des moments discursifs analysés, différentes formes d'allusion ont ainsi émergé : non seulement celles auxquelles s'est intéressée Authier-Revuz (2000), l'allusion aux mots de l'autre, mais également celles qui découlent du sens commun de l'allusion, et qu'on retrouve dans les dictionnaires d'usage: "manière d'éveiller l'idée d'une personne ou d'une chose sans en faire expressément mention " (le Petit Robert). Je discuterai ici des formes de mémoire que semblent inscrire trois catégories d'allusion, exemplifiées ici à titre d'exemple « pour réfléchir». 
26 Il y a, comme le dit Authier-Revuz, des allusions qui inscrivent les mots de l'autre: allusion à des mots donc, mais également à des constructions, des consonances, des formulations déjà énoncées par d'autres et dont l'énonciateur ne mentionne pas l'origine, soit parce qu'il ne le veut pas (allusion voulue) soit parce qu'il n'a pas conscience qu'il s'agit d'un dire emprunté (allusion subie). Quoiqu'il en soit, ces allusions s'inscrivent dans le fil horizontal du discours et inscrivent de ce fait, de manière plus ou moins clandestine, d'autres discours, parfois autant de fils verticaux que d'allusions.

Ces allusions surgissent dans les titres de certains journaux, en particulier à la une ou dans le péritexte des articles de commentaire, mais également au fil des ces textes (éditoriaux, chroniques, points de vue, dessins de presse, etc.) ainsi que dans les dires, situés et cités entre guillemets, des textes d'information (ex. 15 infra) :

5. L'horreur alimentaire

6. «Inutile de sauter comme un cabri / L'Europe il faut la faire »

(France-Inter, 5 décembre 2004)

7. Fracture urbaine / Désintégration sociale

8. Rupture tranquille

Dans l'exemple 5, le titre de l'éditorial, qui porte sur l'affaire des farines alimentaires, « rappelle» le titre d'un ouvrage de Viviane Forrester, L'horreur économique: on peut ici faire l'hypothèse que l'allusion est "voulue ", comme le dit J. Authier; elle est aussi « risquée » (ibidem) car il n'est pas dit que tous les lecteurs la perçoivent... De même, le titre de la une Alerte au soja fou (ex 1), testé auprès d'étudiants, évoquait pour tous l'affaire de la vache folle (désignation qui, dans les médias français, avait peu à peu supplanté la dénomination Encéphalopathie Spongiforme Bovine ou ESB) et pour certains seulement la structure et les sons de l'émission télévisée Alerte à Malibu.

De même on peut ressentir à l'écoute de l'énoncé 6 qu'il y a « comme quelque chose qu'on aurait déjà entendu ", même si on a oublié la phrase d'origine et qui l'a dite ${ }^{4}$, et lorsque Corinne Lepage, écologiste, dit, lors d'un entretien à la radio, qu'elle n'est pas «un faucheur volontaire » (ex.4), elle reprend sans marque particulière (sinon le un, qui me semble un indice d'altérité énonciative) la désignation que ceux qui détruisent les cultures d'OGM ont choisi de se donner, sans la transformer. Mais ce n'est pas le cas des énoncés 7 et 8 : fracture urbaine et désintégration sociale sont apparus lors de la crise des banlieues de novembre 2005, formés sur « la fracture sociale », thème d'une campagne de Chirac, et sur le principe de "l'intégration sociale». Et si le slogan d'une affiche de la campagne de Mitterrand de 1981, «la force tranquille », avait été repris à l'identique dans un tract de Le Pen en $2002^{5}$, la rupture tranquille, annoncée en 2007 par Sarkozy (ex. 8), semble bien dériver du slogan initial.

On a donc bien là une forme d'intertextualité intentionnelle, mais aussi une forme d'appel à "quelque chose », qui peut être de l'ordre du «vague», et qui serait stocké dans la mémoire du lecteur, sans quoi l'allusion ne peut être "sentie » et reste sans « effet»: mémorisation antérieure de formes, de sons, de sens, et même de constructions lorsque « poulet à la dioxine » rappelle à certains la chanson de Jean Ferrat des années 1970, où il était question des poulets aux hormones qu'on mangeait dans les HLM. Dans ces rappels, qui invitent le lecteur à se "remémorer ", des effets de sens sont donc constitutivement associés à des effets de mémoire.

31 Il y a d'autre part inscrit en sus dans Alerte au soja fou le rappel de la crise de la vache folle, c'est-à-dire d'un événement de la même famille, à laquelle est associée au fil du temps la notion de risque lié à l'alimentation. C'est ainsi que va jouer dans cet appel à la mémoire 
que constitue un mot-événement un ensemble de représentations mentales qui lui sont associées. Il en est ainsi de vache folle dans l'exemple 1, qui ne désigne évidemment pas un animal au comportement anormal, comme d'ailleurs Bhopal ou Tchernobyl ne désignent plus au fil du discours les villes du même nom mais les catastrophes chimiques ou nucléaires qui s'y sont produites (ex. 9,10,11, 18 infra).

Au fil d'un titre, du déroulement d'un texte, à l'intérieur d'une citation, certains mots «éveillent » le rappel d'un fait ou d'un événement. Il y a donc bien au préalable stockage dans la mémoire sémantique (dite parfois encyclopédique) du mot et de ce qu'il évoque. C'est là que le débat s'engage : qu'est-ce que la mémoire individuelle stocke exactement ? des discours ou des images? des mots et des images mentales? Car le 11 septembre (autre mot-événement souvent rencontré avec ses cotextes depuis..., après...) ne renvoie-t-il pas au moins autant aux images des tours qui tombent, que les télévisions ont repassées en boucle, qu'aux conséquences de cet événement ? De même pour le tsunami de décembre 2004 en Asie: les images télévisées, les photos dans la presse et les récits qui les accompagnent finissent par faire stocker ces événements avec les émotions que la représentation médiatique suscite.

Les mots-événements ont des effets de mémoire qui dépassent en effet le sens des mots : ils véhiculent des représentations, voire des émotions, liées à des connaissances, à des faits et à des savoirs plutôt qu'à des dires. Ils sembleraient donc liés à la mémoire épisodique, en particulier lorsqu'il s'agit d'événements physiques liés à des expériences, réellement vécues ou par images interposées ${ }^{6}$, que J.H. Searle 1995 distingue à juste titre des événements sociaux. Mais ils peuvent également renvoyer à des savoirs historiques et fonctionner par analogie : lorsque la Grande Bretagne a été désignée pour organiser les Jeux olympiques de 2012 devant la France, Libération a titré le lendemain le récit de l'explosion de joie des Britanniques à Londres : Waterloo à Trafalgar Square.

Une particularité de ces mots-événements, c'est qu'ils peuvent être précédés de désignations catégorisantes, voire qualifiantes, qui contribuent à leur donner un air de famille: affaire, scandale, crise, fléau pour la famille des événements scientifiques à caractère politique; saga, feuilleton pour les OGM; et le paradigme métaphorique de la guerre pour désigner les controverses autour des OGM ainsi que les actes à répétition des anti-OGM : bataille, guerre; croisade ; fronde, résistance...

Une autre particularité, c'est que, au fil du discours, certaines constructions contribuent à relier ces événements, dans une série marquée par une temporalité à court terme qui va contribuer à leur imprimer un sens social :

9. De Tchernobyl au sida en passant par le sang contaminé et la maladie de Creutzfeld-Jakob, la mondialisation se manifeste sous la forme la plus effrayante qui soit, celle de la contamination (Libération)

10. Après la «vache folle » britannique et la dioxine belge, le scandale des farines animales montre, une nouvelle fois, que, pour faire face à l'horreur alimentaire, nous avons plus que jamais besoin d'un Etat fort (le Monde)

11. [...] cette affaire en rappelle fâcheusement d'autres de sinistre mémoire : la dissimulation des risques d'irradiation après la catastrophe de Tchernobyl, l'affaire du sang contaminé et celle de la vache folle (le Figaro)

Les rapprochements opérés contribuent à construire une mémoire de ces événements, une mémoire partagée, autour de la notion de risque et des notions qui lui sont actuellement associées (le principe de précaution, la transparence, la traçabilité - voir Moirand 2003 a et b), car «si les souvenirs reparaissent, c'est que la société, à chaque instant, dispose de moyens nécessaires pour les reproduire» (Halbwachs 1994 [1925]: 
290) et que «durant le cours de ma vie, le groupe national dont je fais partie a été le théâtre d'un certain nombre d'événements dont je dis que je me souviens, mais que je n'ai connus que par les journaux ou les témoignages de ceux qui y furent directement mêlés » (Halbwachs 1997 [1950] : 98).

On voit ainsi s'inscrire au fil du discours non seulement le mot-événement, mais ce qui découle des représentations qu'on lui a mentalement associées (des mots, des images, des sons) ainsi que la temporalité qui lui est attachée, voire des domaines de mémoire également associés. Ainsi, évoquer un Tchernobyl aviaire (ex. 2), c'est provoquer une association entre le nuage de pollution nucléaire qui dépassa les frontières de l'URSS et le danger de propagation du virus de la grippe aviaire, mais c'est aussi mettre le doigt sur la contradiction entre les discours tenus à l'époque sur l'absence de ce nuage au-dessus de la France et les discours actuels qui montrent qu'il n'en était rien et qui dénoncent aujourd'hui les conséquences de son passage sur l'Est de la France.

Il y a enfin, inscrits au fil du discours, des segments discursifs qui ne renvoient pas à des dires réellement énoncés ou à des paroles concrètes.

Des titres comme L'OGM ou la faim? (ex. 3) ou Les OGM comme solution à la faim dans le monde? condensent en peu de mots l'argument des pro-OGM, tandis que la forme interrogative inscrit la méfiance des anti-OGM. Ils ont à la fois une fonction de rappel ou d'information tout en soulignant l'existence d'une controverse. Des formes comme l'interrogation, la thématisation, la négation, la nominalisation, l'incise, etc. sont ainsi des lieux d'inscription du déjà-dit (Bres 1998) et renvoient souvent « à du discursif qui se perd dans la nuit des temps et que nous avons toujours su !» (Maldidier 1993 : 114).

Sont ainsi inscrits des dires qui ne renvoient pas à des énonciateurs situés, à des situations antérieures concrètes, à des discours précis, mais à des positionnements énonciatifs ou à des arguments (c'est moi qui souligne en italiques) :

12. L'arrivée sur le marché européen du premier aliment génétiquement modifié montre que la leçon de la crise de la vache folle - on ne joue pas impunément avec la nature - n'a pas encore été tirée par l'Union européenne

13. Ce qu'on appelle aujourd'hui manipulation [...] en des temps plus optimistes s'appelait tout simplement progrès

14. Dans cette nouvelle boîte de Pandore, on peut trouver aussi bien une corne d'abondance (le plus vieux rêve de l'humanité : une nourriture saine, goûteuse et bon marché pour tous) ou de possibles fléaux (Frankenstein s'est échappé déguisé en marchand de pop-corn)

41 Mais dès qu'on se place dans l'énoncé suivi, on perçoit combien il devient difficile de distinguer entre les inscriptions (conscientes ou non) de dires antérieurs et des mots de l'autre et celles qui font appel à la mémoire de faits ou d'événements. On perçoit également la complexité des empilements de traits sémantiques (dans fou ou folle, par exemple, ou dans totalitaire) et des emboîtements de discours antérieurs ou des superpositions de faits antérieurs dans le discours présent, dans fléau par exemple, qui rappelle la peste, mais également le sida et autres fléaux « modernes » (dans les ex. 16, 17, 18 , c'est nous qui soulignons en italiques) :

15. "terroristes ", " obscurantisme ", " démarche totalitaire ». Les mots n'étaient pas assez durs, sous la plume du directeur général du groupe Limagrain, pour condamner les destructions de trois parcelles de maïs transgénique [...]

16. Grippe aviaire

Un fléau de plus en Afrique

17. OGM : L’Europe résiste 
Les Européens disent aussi non aux OGM

18. Les crimes sanitaires se succèdent; Il y eut la vache folle et la fièvre aphteuse. Aujourd'hui ce sont les oiseaux migrateurs et leurs frères domestiques qui portent la menace : le virus animal qui, s'il se combinait au virus humain, pourrait provoquer une épidémie redoutable, comparable à la grippe espagnole au début du siècle dernier (la Croix, 17 octobre 2005)

On s'interroge alors sur la façon dont le genre (un éditorial dans l'ex.18), en faisant appel à des domaines de mémoire relevant tour à tour d'une antériorité proche ou lointaine, articule et répartit ces allusions de nature différente au fil du discours présent, et donc sur les formes de mémoire auxquelles font appel l'ordre du discours et les effets mémoriels qu'il produit (voir en 3 infra).

\subsection{Quelles mémoires ? Éléments pour un débat}

43 Le mot mémoire est polysémique. On ne reviendra pas sur cette longue histoire de la mémoire en tant que faculté humaine, qui hante depuis longtemps ceux qui s'intéressent au langage, à la littérature, à la philosophie, à l'histoire, et à ce qui sous-tend la conservation et la transmission des cultures et des savoirs ${ }^{7}$. On s'interrogera en revanche sur son fonctionnement dans les matérialités discursives et en particulier dans les discours médiatiques qu'on a étudiés.

Le mot mémoire réfère autant à la faculté d'acquérir et de stocker des souvenirs qu'à l'opération de restitution des souvenirs ainsi qu'à l'ensemble des données que l'on a justement stockées «en mémoire». Or, comme le dit Denis Mazzuchetti (2003), jeune historien venu aux sciences du langage, si le discours, acte de langage, est permis, supporté par la mémoire (ce qui permet de relier sans doute l'acte de production aux prédiscours - voir Paveau ici même), le discours suscite en retour une activité mémorielle intense, non seulement lors de la compréhension et de l'interprétation mais parce que le discours s'inscrit dans la circularité constitutive et ininterrompue de la parole :

«Toute énonciation, quelque signifiante et complète qu'elle soit par elle-même, ne constitue qu'une fraction d'un courant de communication verbale ininterrompue (touchant à la vie quotidienne, la littérature, la connaissance, la politique, etc.). Mais cette communication verbale ininterrompue ne constitue à son tour qu'un élément de l'évolution tous azimuts et ininterrompue d'un groupe social donné » (Bakhtine/ Volochinov 1977 : 136)

Ainsi le commentaire médiatique repose-t-il sur des indices déclencheurs de souvenirs, souvenirs de dires, de savoirs, d'événements et de représentations tels qu'ils sont reconstruits dans la mémoire de ceux qui le produisent et de ceux qui y sont exposés. Et comme il s'adresse à une pluralité de destinataires et qu'il inscrit en lui-même plusieurs "épaisseurs dialogiques » venues d'une pluralité de discours autres, il présuppose une forme de mémoire collective en même temps qu'il contribue à la construire. Or, si l'on souscrit aux thèses de $\mathrm{M}$. Halbwachs, pour qui «ce sont les autres qui font que les souvenirs nous reviennent » (1994 [1925] : VI), on imagine le rôle joué aujourd'hui par les médias auxquels on est inévitablement exposé. Les discours des médias constituent à la fois un catalyseur de la remontée des souvenirs enfouis et un élément moteur de la construction d'une mémoire interdiscursive, sans exclure un autre rôle, qui n'est pas contradictoire : celui d'être un censeur de savoirs qu'on «oublie », volontairement ou non, de « faire remonter ». 

langage, des sciences cognitives et des sciences humaines (histoire, psychologie, société... ). Elle aurait de fait un triple ancrage, discursif en premier lieu, et donc historique, par les traces de ses propres inscriptions, mais également cognitif (pour ce qui est de la mémoire individuelle) et sociale (pour la part qui lui vient de la mémoire collective). Faculté psycho-cognitive, elle permet à un sujet de se remémorer un énoncé et de l'inscrire dans son discours, mais aussi de le reconnaître et de l'associer à d'autres énoncés ou d'autres images stockées en mémoire. Le discours étant par ailleurs constitué de répétitions (de formes, de sens, de représentations), la mémoire constitutive du discours est tributaire de la langue et donc de ses ressources formelles et associatives (ce qu'on l'on trouvait chez Saussure dans sa conception des rapports associatifs et que l'on retrouve chez les spécialistes des constructions figées, G. Gross par exemple - voir Mazzucchetti 2003).

Je ne discuterai ici que des formes de l'allusion traitées supra dans leurs rapports avec la mémoire. L'allusion permet en effet le rappel des dires, des faits et des événements, et donc de domaines de mémoire et de savoirs : les traces d'allusion fonctionnent de ce fait comme autant d'indices de contextualisation (Moirand 2000, 2004a). Et si l'on pose (comme on le fera en 3) que la répartition de ces indices au fil du discours contribue à l'orientation pragmatique $\mathrm{du}$ texte, on fait une place au traitement $\operatorname{cognitif}^{8}$ qui intervient dans la compréhension et l'interprétation des énoncés.

L'acte de nommer entrevu supra (en 1.2.), lorsqu'il sert à désigner et à caractériser des événements et des acteurs, contribue par l'utilisation de mots empruntés à des moments de l'histoire à rappeler les domaines de mémoire qui leur sont attachés. Ce serait la mémoire sémantique qui intervient ici lorsque croisade ou fronde désignent par exemple les actions des acteurs (dans croisade anti-maïs transgénique), et faucheurs volontaires les acteurs eux-mêmes quelques temps plus tard. Si croisade ou fronde nous replacent dans l'histoire ancienne, le premier évoque également le discours de Bush après le 11 septembre, et faucheur volontaire (qui évoque la désobéissance civile) a été proposé par les acteurs eux-mêmes face aux désignations que leur donnaient leurs opposants (par exemple le mot vandale, utilisé par des scientifiques, des ministres, des responsables de firmes agro-alimentaires, des journalistes - voir également l'ex. 15). discours, à travers les différentes facettes que les désignations construisent de ces objets, en les comparant ou en les enrichissant des connaissances stockées antérieurement, que l'on accède à celles-ci de façon automatique ou volontairement. Qu'il y ait ou non détournement du sens original, amalgame «voulu» ou «subi» lorsque qu'on compare « les nouveaux vandales » que seraient les destructeurs de plants d'OGM à ceux « qui, sous la Terreur, détruisaient, saccageaient, profanaient les "monuments des arts et de sciences" " (le Monde), ou lorsque sont rapportés par les médias les paroles des autorités de l'Éducation nationale, traitant de «trotskystes» les acteurs de l'occupation de la Sorbonne, on voit que le mot fonctionne comme un rappel de connaissances stockées en mémoire : «les hommes vivant en société usent de mots dont ils comprennent le sens : c'est la condition de la pensée collective. Or chaque mot (compris) s'accompagne de souvenirs, et il n'y a pas de souvenirs auxquels nous ne puissions faire correspondre des mots » (Halbwachs 1994[1925] : 279).

On peut s'interroger sur les domaines de mémoire que certains mots font ainsi émerger : faisant appel à ceux d'une mémoire collective certes dans ses rapports à l'histoire ancienne ou à l'histoire récente de la France, le rappel fonctionne différemment selon les 
souvenirs emmagasinés par chacun, selon les situations ou les discours où on a rencontré le mot, ce qui nous ramène à l'ancrage discursif de la mémoire. L'inscription discursive d'une désignation ou d'une formulation n'appelle pas en mémoire les mêmes souvenirs pour tous, comme l'avait bien montré Halbwachs en distinguant les rôles des mémoires collectives de la mémoire individuelle (voir infra).

Ce sont cependant les allusions à des connaissances supposées partagées, dont l'ancrage discursif paraissait moins évident, qui nous a fait peu à peu douter du caractère strictement discursif de la mémoire interdiscursive : celles évoquées plus haut dans l'ex. 14 à travers des mots comme Frankenstein ou Pandore, avec ou sans sa boîte.... Allusions à des savoirs de nature encyclopédique, plutôt qu'à des connaissances qui seraient partagées, elles semblent en raison de leur fréquence faire appel à des images stéréotypées dont on a oublié l'histoire: des tests auprès d'étudiants de doctorat ont montré à quel point les connaissances étaient vagues sur l'histoire de ces allusions (seuls les étudiants grecs connaissaient l'histoire de Pandore). Mais cette méconnaissance ne nuisait pas à la compréhension de l'argumentation, parce qu'il suffit de retenir ici au fil du temps les valeurs attachées à ces expressions, par exemple une valeur négative dans nourriture Frankenstein, et par suite dans le pop corn qui y est associé dans l'ex. 14.

Ce sont enfin les mots-événements qui ont conduit à rediscuter de l'ancrage cognitif de la mémoire (ex. 9 sq. et ex. 19). "Sang contaminé », «Tchernobyl ", « Mai 68 ", "grippe espagnole », «la Terreur » font bien remonter en mémoire des faits et des événements plus ou moins récents. Ils posent la question du rapport entre les traits sémantiques inscrits dans les mots et les images ou les visualisations de l'événement tel qu'il est reconstruit en mémoire, avec (ou sans) les discours qui y sont attachés. De ce fait, si la mémoire permet de contextualiser des informations issues de la mémoire sémantique et d'enregistrer le souvenir précis d'un épisode passé, qu'on l'ait ou non vécu directement, on peut admettre qu'il y aurait ici une place pour une intervention de ce qu'on appelle la mémoire épisodique, avec la mémoire sémantique ou à côté d'elle, mémoire reliée pour certains faits à des émotions. Mais si la question posée aux linguistes reste celle de leur "stabilisation » et de leur «construction » sémantique (comment et à partir de quand « sang contaminé » finit par désigner l'affaire du sang contaminé ?), la question que je me pose aujourd'hui, c'est plutôt celle de leurs relations à la mémoire.

Je ne pense plus qu'on puisse attribuer à la seule interdiscursivité l'allusion à des faits ou à des événements au travers des mots ou des expressions qui les désignent. Précédés d'un déterminant défini, ces mots-événements correspondent à des opérations de référence et finissent par fonctionner comme des dénominations partagées, qui renvoient à un ensemble complexe de faits, de visualisations et de discours. Lorsqu'il s'agit d'événements du monde physique (cyclones, tremblements de terre, tempêtes), des représentations de l'ordre du visuel ou même des sensations expériencielles surgissent ainsi en mémoire à l'évocation du mot-événement. Mémoire épisodique et mémoire sémantique interviendraient alors, à côté du discours, à la fois dans la construction et l'intervention d'une mémoire collective, qui n'est pas la même pour tous les groupes sociaux selon le degré d'implication dans l'événement: dans un séminaire au Chili, pendant l'été 2005, l'allusion au 11 septembre n'évoquait pas en premier le 11 septembre 2001 mais bien le coup d'État du 11 septembre 1973 au Chili, que la plupart des étudiants présents n'avaient pourtant pas eux-mêmes vécu, mais dont on voyait encore des traces « physiques » sur les murs de certains bâtiments de Valparaiso. 
54 allusions disséminées au fil des textes, c'est que celles-ci contribuent à renforcer les mémoires collectives communes de certaines communautés langagières, mais qu'elles participent également à la construction de la mémoire individuelle que chacun construit à travers son trajet d'expériences humaines, trajet qui n'est pas fait seulement d'expositions au discours mais qui est partiellement mémorisé à travers du discours : «si la mémoire collective tire sa force et sa durée de ce qu'elle a pour support un ensemble d'hommes, ce sont cependant des individus qui se souviennent, en tant que membres du groupe. De cette masse de souvenirs communs, et qui s'appuient l'un sur l'autre, ce ne sont pas les mêmes qui apparaîtront avec le plus d'intensité à chacun d'eux » (Halbwachs 1997 [1950] : 94-95). Mais comme le dit par ailleurs Maurice Halbwachs (ibidem : 98), «le fonctionnement de la mémoire individuelle n'est pas possible sans ses instruments que sont les mots et les idées, que l'individu n'a pas inventés, et qu'il emprunte à son milieu ", à travers les discours et les images auxquels il est exposé, pourrions-nous ajouter.

À travers les médias, il semble se construire une mémoire collective médiatique, majoritairement interdiscursive mais pas totalement, qui fait appel aux mémoires sémantiques et épisodiques des individus tout en contribuant à les transformer, et le fil du discours contribue à cette construction à travers les relations qu'il permet entre les allusions disséminées au fil du discours, et la façon dont il les associe ou les relie.

\section{Retour à l'ordre du discours}

Si les allusions inscrivent des dires, des faits, des représentations « extérieurs » au texte qui se déroule, c'est leur répartition au fil du discours qui contribue à l'orientation pragmatique des genres du commentaire. Ainsi l'explication ou l'argumentation des éditoriaux fait une utilisation particulière des allusions qui interviennent dans l'éclairage qui est donné aux faits, aux dires, aux événements, et la lecture et l'interprétation des textes de commentaire suppose une activité cognitive qui permette de comprendre cet éclairage. Quelles mémoires interviennent au fil du texte dans la construction et la compréhension du fil du discours? Tenter de répondre à cette question m'a conduite à articuler aux notions d'interdiscours et de dialogisme les notions de schématisation, d'éclairage et d'objet de discours du modèle proposé par Grize (1978, 1996, 2004, par exemple), parce qu'il s'agit d'une approche cognitive de l'argumentation, qui repose sur la notion de représentation.

Pour Grize, tout discours construit une sorte de micro-univers à destination de son auditoire, c'est-à-dire qu'il ne dit pas « tout ». Le locuteur (A) propose à son interlocuteur (B) une schématisation, c'est-à-dire une reconstruction du monde qui ne reflète pas les exigences d'une observation scientifique mais la façon dont A se représente la situation: la schématisation est ainsi construite en fonction de la finalité de $\mathrm{A}$, mais aussi des représentations que $\mathrm{A}$ se fait de $\mathrm{B}$, de celles qu'il a de dont il parle (le thème $\mathrm{T}$, qui se décline au travers des objets de discours représentés) et de celles qu'il veut donner de luimême. On peut inférer les représentations des images qui sont proposées dans la matérialité discursive et qui constituent les traces d'une pensée "mise en mots». Ce modèle global permet de relier les traces des opérations langagières et d'étudier l'effet produit par leur répartition et leur combinaison au fil du texte. 
58 L'observation des discours de presse que j'ai alors développée (Moirand 2007a pour une synthèse) intègre, au niveau local, le repérage des traces des opérations de référence, de prédication et d'énonciation, et prend en compte au niveau global le fil du discours avec ses caractéristiques intratextuelles (comme l'inscription et la transformation des objets de discours au travers des phénomènes de reprise et de thématisation - voir Sitri 2003) et ses caractéristiques intertextuelles (discours cités et situés) et interdiscursives (celles qui renvoient à l'histoire et à la mémoire, et en particulier, les différentes formes de l'allusion - voir Moirand 2004a, 2006a et 2007b). On se limitera ici à une réflexion sur le rôle des allusions décrites en 2.1. dans l'orientation pragmatique des énoncés et dans leurs relations avec les formes de la mémoire.

Une première réflexion a découlé des sous-corpus qu'on avait constitués autour des séries de mots-événements telles qu'elles apparaissent dans les exemples 9, 10,11. Le fait de relier par des constructions (de $X \grave{a} Y$, après $X$ et $Y$ ou une simple énumération) des motsévénements (traces d'opérations de référence et de nomination), accompagnés ou non de désignations qualifiantes, créent des associations entre des faits qui, scientifiquement, n'ont rien en commun, et ce sont ces associations qui contribuent à construire une mémoire collective du risque (alimentaire, chimique, écologique...). Il est vraisemblable que la mémoire de travail intervient localement dans la construction de liens entre ces événements déjà présents dans la mémoire sémantique, mais que les listes et les chronologies que les formes construisent, de même que les formes de ces désignations seront à leur tour stockées en mémoire sémantique, si ce n'est déjà fait, et leur mémorisation en tout cas renforcée.

60 Ce que l'on voudrait montrer ici, c'est le rôle que jouent les allusions dans l'éclairage que l'énonciateur donne aux objets du discours qui se déploient dans l'ordre du texte. L'éclairage est une notion issue de la logique naturelle de Grize pour qui «une argumentation ne persuade pas elle-même mais [...] conduit le destinataire à se persuader lui-même » (Grize 2005 : 42-43). Or l'éclairage est donné à travers le choix qui est fait des mots et des constructions lors des actes de nomination, de caractérisation, et d'évaluation de l'énonciateur et participe ainsi à la schématisation construite au fil du texte. Il peut intervenir dès le titre comme dans l'exemple suivant :

\section{UN PETIT MAI-68 DES BANLIEUES}

61 Ce titre, apparu en pleine crise des banlieues (événement de l'automne 2005), donc avant les manifestations étudiantes de 2006 qui ont déclenché la reprise de nombreuses séquences filmées en mai 1968 à la télévision, repose sur le mot-événement "Mai-68 ", allusion qui renvoie à ce qui est stocké dans la mémoire sémantique sur les événements de mai 1968, en particulier au Quartier latin. Mais le mot-événement est ici éclairé par deux éléments du cotexte: le "un » transforme la désignation en caractérisation et " petit » est une autre caractérisation qui minimise l'importance de l'événement présent; l'ensemble « un petit Mai-68 » ainsi rapporté à l'événement présent est alors situé : dans « les banlieues ». On peut ici supposer l'intervention conjointe de la mémoire de travail et de la mémoire sémantique dans l'effet qui est construit et dans l'interprétation que l'on peut faire : le titre, qui apparait dans la page Analyses du Monde et qui est accompagné d'une autodésignation elle aussi éclairante ("Chronique politique»), permet d'anticiper sur la lecture du texte. Les titres apportent souvent un éclairage aux textes qui les suivent, comme on a pu déjà l'entrevoir dans les exemples 16 et $17:$ l'allusion introduite à travers les mots de plus (= il y a d'autres fléaux) ou aussi (les Français et les Néerlandais viennent de dire non au projet de constitution européenne) contribuent à l'éclairage, qui guide 
partiellement l'interprétation de l'énoncé (partiellement, car tout dépend de ce qui est stocké et de ce qui est appelé ou rappelé en mémoire chez le lecteur du texte).

$\mathrm{Au}$ fil du discours, les allusions viennent ainsi éclairer de diverses manières le point de vue du scripteur sur des faits qu'il évoque ou des mots et des dires qu'il emprunte. Ainsi au début d'un éditorial du Figaro publié lors de la crise du Contrat Première Embauche, la façon de nommer les acteurs de cet événement contribue à éclairer le point de vue du scripteur :

20. Cette semaine, les conservateurs seront dans la rue, contre le CPE. Car il ne faut pas se fier aux apparences. Ce sont les porteurs de banderoles qui ne veulent rien changer, défendent le statu quo et s'agrippent à un modèle social remarquable par l'exclusion qu'il engendre aux deux bouts de la vie active - jeunes et seniors. Au point que l'on se demande: pour qui roulent-ils au juste, ces militants de l'immobilisme, sinon pour le maintien de la fracture qui caractérise notre marché du travail entre salariés surprotégés et main-d'œuvre surprécarisée ?

[début de l'éditorial : La rue contre les réformes, le Figaro, 13 mars 2006]

Le début du texte éclaire de manière particulière les manifestants:«ce sont eux les conservateurs » («pas les autres, pas nous...»). Mais en les désignant plus loin comme «les porteurs de banderoles ", il leur associe des images visuelles, celles que l'on voit au journal télévisé, tout en donnant un côté dérisoire à leur action (au travers de la description d'une action statique : porter des banderoles) plutôt que d'essayer d'expliquer leur points de vue. L'éclairage est alors renforcé par une désignation, qui associant deux termes contradictoires («militants» et «immobilisme»), contribue à renforcer les valeurs que l'on veut transmettre de même que « conservateurs » et « contre le CPE » font appel à deux domaines de mémoire antagonistes... De manière automatique, les mémoires à court terme (mémoire de travail) et à long terme (sémantique) sont convoquées par cette chaîne coréférentielle qui construit une représentation des manifestants ainsi que par l'usage qui est fait de mots circulants qu'on détourne (sémantiquement et/ou formellement) de leur contexte, donc de leur domaine de mémoire originel (modèle social, fracture, surprotégé/surprécarisé, par exemple). Cela ne fait que confirmer l'hypothèse d'une mémoire interdiscursive qui répéte et reprend les mots de l'autre mais qui ne peut fonctionner (ici dans l'éclairage qui est donné) qu'avec l'intervention (automatique ou volontaire) des mémoires cognitives et des mémoires collectives. Pour moi, la mémoire des mots et des dires contribue à l'éclairage, et « les phénomènes d'éclairage apparaissent comme le résultat de mécanismes plus ou moins consciemment mis en place et qui doivent conduire le lecteur à inférer par lui-même un jugement de valeur » (Grize 1992 : 25). Il y a, il me semble, dans cette ronde des discours qui se déploie dans les médias (et qui rappelle « le courant de communication verbale ininterrompue » dont parle Bakhtine) les traces d'un traitement cognitif des discours qu'on utilise et/ou auxquels on est exposé : les allusions seraient des indices d'actualisation de ce traitement dans la matérialité discursive.

On prendra comme dernier exemple de cette actualisation et de son rôle dans l'orientation argumentative des textes un extrait du texte dont le titre constitue l'exemple 19 et qui trouve ici son explication (car si le titre éclaire le texte, le texte éclaire en retour le titre) :

21 [...] Fin de règne, président contesté dans son camp, émergence d'un favori de la majorité, pour succéder au monarque vieillissant, révolte de jeunes, cela ne rappelle-t-il rien? Bien sûr les différences entre les violences qui ont éclaté dans certaines villes de banlieue et les prodromes des événements de mai 1968 sont 
écrasantes. Les révoltés ne sont pas des étudiants... [fils et petits fils d'immigrés/ souvent chômeurs/il y a parmi eux des voyous inquiétants]

N'y a-t-il pas, pourtant, dans ces émeutes qui semblent ne se répandre que par contagion, sans organisation ni consignes, le ferment possible d'une prise de conscience? Plus de vingt ans après les marches des Beurs, qui avaient exprimé la protestation des jeunes Français d'origine arabe et africaine contre les obstacles mis à leur intégration, on peut considérer les échauffourées de Clichy-sous-bois, Montfermeil, La Courneuve et ailleurs comme une nouvelle preuve de la disparition des repères politiques. Les insurgés d'aujourd'hui n'ont pas d'autre horizon que la répétition de bastons sans autre fonction que d'extérioriser leur mal-être. Cette « canaille »-là, à la différence de celle que chantaient les révolutionnaires de la Commune, n'a ni mémoire ni rêves.

Mais il est possible aussi qu'à leur manière ces révoltés - ou une partie d'entre eux trouvent le chemin d'une revendication et d'une ambition qui les fassent passer $\mathrm{du}$ statut d'objet d'étude sociologique à celui de citoyens.

[le Monde, 5 novembre 2005)

\section{l'événement présent (les échauffourées de au travers d'allusions à des faits c} l'énumération se termine par un appel explicite à la mémoire: "cela ne rappelle-t-il rien? ». Mais l'analogie s'arrête avec la question, dans une concession (bien sûr) qui admet que c'est différent (différences... écrasantes), et que «les révoltés » d'aujourd'hui ne sont pas des étudiants, mais « des fils et petits-fils d'immigrés », « souvent chômeurs » et " parmi eux des voyous inquiétants ».

d'un autre événement qu'on les désigne indirectement («la marche des Beurs », «jeunes Français d'origine arabe et africaine ») avant de construire au fil du texte une autre représentation de ces "révoltés ", à partir d'une autre de leurs facettes: l'éclairage donné dans "les insurgés d'aujourd'hui » conduit à " cette canaille »-là, mot qui renvoie à un domaine de mémoire d'une autre époque et à un événement de l'histoire explicitement nommé, « la Commune », mais dont la forme sonore rappelle « racaille », désignation emblématique de l'événement présent. Le micro-univers qui est construit semble alors opposer à ceux de la Commune les révoltés de 2005, parce qu'ils n'ont "ni mémoire ni rêves ", et sans doute implicitement (ce qui expliquerait le « petit » du titre) à ceux de Mai 68... au lecteur à travers la question posée au début de l'avant dernier paragraphe (y-a-t-il « ... le ferment possible d'une prise de conscience?») et donc un autre éclairage des révoltés actuels : ni canailles/racailles, ni révolutionnaires rêveurs, ils peuvent peut-être (il est possible), pas tous (une partie d'entre eux) devenir citoyens. La représentation qui est construite des acteurs de l'événement passe par une chaîne co-référentielle de désignations, dont chacune porte une mémoire, celle d'événements antérieurs qui appartiennent à l'histoire, récente ou ancienne.

Dans le traitement de cette progression de la schématisation, interviennent de manière partiellement automatique et inconsciente, partiellement intentionnelle, différentes mémoires : la mémoire de travail avec son automatisme lié à la langue, la mémoire des mots et des dires dont on a pas toujours conscience (l'interdiscours), la mémoire des événements tels qu'on les a stockés avec leur histoire (mémoire sémantique, mémoire épisodique), et tels que les communautés auxquelles on appartient s'en souviennent (mémoire collective). 


\section{servent à comprendre les événements physiques et sociaux et que ces représentations} interviennent dans les façons de désigner et de caractériser, voire dans le récit de ces événements. Car c'est le langage qui permet de les classer, de les «repenser » et d'en « reparler » à d'autres : la langue permet de les ranger dans des catégories partagées (les dénominations), mais le discours contribue au partage des points de vue à l'intérieur d'une société, d'un monde social ou d'une communauté langagière ainsi qu'à la mémorisation de ces points de vue. Faute de quoi, il serait impossible de communiquer, et d'agir sur l'autre. Or si les représentations mentales sont à la fois modelées par les bases biologiques du cerveau et sans cesse remodelées par le contexte social avec lequel on interagit, elles le sont aussi au travers des représentations qui sont "données à voir " dans les matérialités discursives ou sémiotiques auxquelles on est exposé (par exemple dans les médias)'.

71 Les allusions pourraient ainsi constituer des traces des représentations que l'énonciateur se fait des univers de croyances, de connaissances ou d'expériences des destinataires, et des traces du traitement cognitif préalable à la production du discours effectué (consciemment ou non) par l'énonciateur, avec ses rappels et ses oublis. Entrant ainsi dans la construction de la réalité physique ou sociale qui est «donnée à voir » par le langage, elles constituent en retour pour les destinataires des signes de rappel des représentations enfouies dans leur espace mental et des indices de contextualisation nécessaires à la compréhension des faits, des événements et des façons de les désigner, de les raconter et de les évaluer. Leur distribution dans l'intratexte contribue par sa capacité à inscrire les interactions entre les discours antérieurs dont elles portent la mémoire et les discours présents à faire appel à des représentations et à des valeurs qui interviennent dans l'orientation pragmatique du texte. La compétence mémorielle reposerait ainsi sur la capacité à mobiliser rapidement les savoirs et les discours en production comme en compréhension, et les allusions fonctionneraient comme des signaux de cette mobilisation (volontaire ou non).

72 Là réside en effet l'intérêt méthodologique d'articuler avec une analyse du discours qui s'appuie sur les traces de la mémoire et les signaux qui y font appel la notion de schématisation de Grize, parce que celle-ci porte en elle, comme le dit A. Berrendonner $1997^{10}$ : « une théorie de la référence et du contexte qui tranche de façon radicale avec un certain chosisme ambiant. Elle suppose en effet qu'au lieu d'assimiler les référents du discours aux realia en se recommandant du bon sens ou de Frege, on leur reconnaisse le statut de représentations cognitives, de schémas mentaux, dotés d'une structure formelle dont la description est affaire de logique et de sémiologie. Quant au contexte, elle conduit à y voir non pas un cadre informationnel et situationnel fixé à titre préalable, mais le produit dynamique de l'activité de communication : un capital évolutif de connaissances, d'hypothèses et d'assomptions partagées, assimilable à une sorte de mémoire collective des interlocuteurs ». 


\section{BIBLIOGRAPHIE}

Adam J.-M. (2004) : « Introduction », dans Adam J.-M., Grize J.-B. et Ali Bouacha M. (éds) : Textes et discours : catégories pour l'analyse, Éditions universitaires de Dijon, 5-19.

Araújo Carreira M. H. (2004) : « La construction discursive de la désignation de l'autre et de soimême ", dans Textes et discours : catégories pour l'analyse, Éditions universitaires de Dijon, 145-162. Authier-Revuz J. (1982) : « Hétérogénéité montrée et hétérogénéité énonciative : éléments pour une approche de l'autre dans le discours ", dans DRLAV, revue de linguistique, 91-151.

Authier-Revuz J. (2000) : « Aux risques de l'allusion », dans L'allusion dans la littérature, Paris, Presses universitaires de Paris Sorbonne, 209-235.

Bakhtine M. (1977, traduction) : Le marxisme et la philosophie du langage, Paris, Éditions de Minuit. Bakhtine M. (1984, traduction) : Esthétique de la création verbale, Paris, Gallimard.

Bres J. (1998) : « Entendre des voix : de quelques marqueurs dialogiques en français », dans Bres J. et alii (éds) : L'autre en discours, Université Paul Valéry - Montpellier 3, Praxiling et Université de Rouen, Dyalang, 191-212.

Charaudeau P. et Maingueneau D. (éds) (2002) : Dictionnaire d'analyse du discours, Paris, Éditions du Seuil.

Courtine, J.-J. (éd) (1981) : Analyse du discours politique, Langages 62.

Courtine J.-J. (1989) : Corps et discours : éléments d'histoire des pratiques langagières, Dossier de présentation pour l'obtention d'une thèse d'État sur travaux, Université Paris 10 - Nanterre.

Denhière G. (éd.) (1984) : Il était une fois... Compréhension et souvenirs de récits, Presses universitaires de Lille.

Détrie C., Siblot P. et Verine B. (éds) (2001) :Termes et concepts pour l'analyse du discours. Une approche praxématique, Paris, Champion.

Dijk van T.A. (2006) : « Politique, idéologie et discours »,dans SEMEN 21, 73-102.

Dijk van T.A. et Kintsch W. (1983) : Strategies of Discourse Comprehension. New York, Academic Press Inc.

Grice H.P. (1979, traduction) : « Logique et conversation», dans Communications 30, 57-72.

Grize J.-B. (1978) : « Schématisations, représentations et images », dans Stratégies discursives, Presses universitaires de Lyon, 45-52.

Grize J.-B. (1992). «Éclairage », dans Grize J.-B. : Un signe parmi d'autres, Hauterive, Suisse, Éditions Gilles Attinger, 22-25.

Grize J.-B. (1996) : Logique naturelle et communications. Paris, Presses Universitaires de France. Grize (2004) : «Argumentation et logique naturelle », dans Textes et discours : catégories pour l'analyse, Éditions universitaire de Dijon, 23-27.

Grize J.-B. (2005) : « Le point de vue de la logique naturelle » dans Doury M. et Moirand S. : L'argumentation aujourd'hui. Positions théoriques en confrontation, Paris, Presses Sorbonne Nouvelle, 35-43. 
Grunig, B.-N. (2005) : « Lexique et visualisation », dans Linx 52, 43-48.

Halbwachs M. (1994 [1925]) : Les cadres sociaux de la mémoire, Paris, Albin Michel.

Halbwachs M. (1997 [1950]) : La mémoire collective, Paris, Albin Michel.

Kintsch W. (1974) : The Representation of Meaning in Memory, Hillsdale, N.J., Lawrence Erlbaum.

Kintsch W. et van Dijk T.A. (1975) : « Comment on se rappelle et on résume des histoires », dans Langages 40.

Lecomte A. (1981) : « Comment Einstein raconte comment Newton expliquait la lumière (ou le rôle de la mémoire interdiscursive dans le processus explicatif) ", dans Revue européenne des sciences sociales et Cahiers Vilfredo Pareto XIX 56, 69-93.

Le Goff J. (1988) : Histoire et mémoire. Paris, Gallimard.

Lemardeley M.-C., Bonafous-Murat C. et Topia A. (2006) : Mémoires perdues, mémoires vives. Paris, Presses Sorbonne Nouvelle.

Maldidier, D. (1990) : L'inquiétude du discours, Textes de Michel Pêcheux choisis et présentés, Paris, Éditions des Cendres.

Maldidier, D. (1993) : «L'inquiétude du discours. Un trajet dans l'histoire de l'analyse du discours : le travail de Michel Pêcheux », dans SEMEN 9, 107-119.

Mazzucchetti D. (2003) : La mémoire discursive, dossier présenté en séminaire de DEA, Université Sorbonne nouvelle - Paris 3.

Micheli R. (2006) : « Contexte et contextualisation en analyse du discours : regard sur les traces de T. van Dijk », dans SEMEN 21, 103-120.

Moirand, S. (2000) : «Les indices dialogiques de contextualisation dans la presse ordinaire » dans Cahiers de praxématique $33,145-184$.

Moirand S. (2001) : " Le choc des sens inscrits dans la mémoire des mots », exposé présenté au séminaire de l'École doctorale Sciences du langage, Université Paul Valéry - Montpellier 3, document de travail électronique.

Moirand S. (2003a) : « Les lieux d'inscription d'une mémoire interdiscursive », dans Härmä J. : Le langage des médias : des discours éphémères ?, Paris, l'Harmattan, 83-111.

Moirand S. (2003b): « Communicative and Cognitive Dimensions of Discourse on Science in the French Mass Media », dans Discourse Studies 5-2, 175-206.

Moirand, S. (2004a) : « Le texte et ses contextes », dans Texte et discours : catégories pour l'analyse, Éditions universitaires de Dijon, 129-143.

Moirand, S. (2004b) : L'impossible clôture des corpus médiatiques ou la construction des observables entre catégorisation et contextualisation, dans TRANEL 40, 71-92.

Moirand, S. (2004c) : « Nomination, caractérisation et objet de discours : questionnements autour du dialogisme et de la mémoire des mots ", dans Cassanas, A., Demange A., Laurent B. et Leclerc, A. (éds) : Dialogisme et nomination, Publications de l'université Paul Valéry - Montpellier 3, 27-64.

Moirand, S (2005) : «Le dialogisme, entre problématiques énonciatives et théories discursives », dans Cahiers de praxématique 43, 189-220.

Moirand S. (2006a) : « Responsabilité et énonciation dans la presse quotidienne :

questionnements sur les observables et les catégories d'analyse ", dans SEMEN 22, 45-60. 
Moirand S. (2006b) « Le choc des discours dans la presse française : l'exemple des violences urbaines et des manifestations étudiantes ", conférence d'ouverture du Colloque international de la FATFA, Adelaide, Australia, 7-9 juillet, à paraître.

Moirand S. (2007a) : Les discours de la presse quotidienne. Observer, analyser, comprendre, Paris, Presses Universitaires de France.

Moirand S. (2007b) « Entre discours et mémoire : le dialogisme à l'épreuve de la presse ordinaire », dans TRANEL 44 : Intertextualité et interdiscursivité dans les médias, sous presse.

Nora P. (éd.) $(1984,1986,1992)$ : Les lieux de mémoire, la République, la Nation, les France, Paris, Gallimard, 7 vol.

Nyckees V. (1998a) : La sémantique, Paris, Belin.

Paveau, M.-A. (2006) : Les prédiscours. Sens, mémoire, cognition, Paris, Presses Sorbonne Nouvelle.

Pêcheux M. (éd) (1975) : Analyse du discours, langue et idéologies, Langages 37.

Petit L. (2006) : La mémoire, Paris, PUF, « Que sais-je ?».

Pottier B. (éd.) (1973) : Le langage, Paris, Centre d'Étude et de Promotion de la Lecture.

Rakotonoelina, F. (2000) : Un modèle d'analyse linguistique des représentations des cultures à partir des textes de la culture informatique (presse et manuels), thèse pour le doctorat en sciences du langage, université Sorbonne nouvelle - Paris 3, Cediscor - Syled.

Rastier F. (1998) : « Le problème épistémologique du contexte et le statut de l'interprétation dans les sciences du langage », dans Langages 129, 97-111.

Reboul, A. et Moeschler J. (2000) : « Pourquoi l'analyse du discours a-t-elle besoin d'une théorie de l'esprit »,dans Berthoud A.-C. et Mondada L. : Modèles du discours en confrontation, Berne, Peter Lang, 185-204.

Ricœur P. (2000) : La mémoire, l'histoire, l'oubli, Paris, Éditions du Seuil.

Searle J.H. (1995) : The Construction of Social Reality, New York, Free Press.

Sciences Humaines 107 (2000) : Souvenirs et mémoires.

Sciences Humaines 128 (2002) : Les représentations mentales.

Siblot P. (1997) :« Nomination et production de sens : le praxème », dans Langages 127, 38-55.

Siblot, P. (2001) : « De la dénomination à la nomination », dans Cahiers de praxématique 36, 189-214. Sperber D.et Wilson D. (1995) : Relevance, Communication and Cognition, Oxford, Basil Blackwell, $2^{\mathrm{e}}$ édition.

Tadié J.-Y. et Tadié J.-M. (1999) : Le sens de la mémoire, Paris, Gallimard.

Tiberghien G. (1997) : La mémoire oubliée, Bruxelles, Mardaga.

Todorov T. (1981) : Mikhail Bakhtine, le principe dialogique suivi des Écrits du cercle de Bakhtine, Paris, Seuil.

Tulving E. (1995) : « Organization of Memory : quo vadis ? », dans Gazzaniga M.S. : The Cognitive Neurosciences, MIT Press.

Volochivov, V.N. (1981). « Le discours dans la vie et le discours dans la poésie », « La structure de l'énoncé », dans Todorov T. : Mikhail Bakhtine, le principe dialogique, 181-215 et 287-314. 


\section{NOTES}

1. L'entrée cognition est cependant présente dans le Dictionnaire des termes et des concepts de l'analyse $d u$ discours, ce qui tient à l'orientation praxématique de ce dictionnaire (et à la conception proposée, entre autres, dans cette approche à l'acte de nomination, dont on reparlera infra): "Ce terme renvoie aux activités et structures psychologiques qui, chez l'humain, recueillent, traitent et communiquent l'information apportée par le monde [...]» (Détrie, Siblot et Verine, éds $2001: 55)$.

2. Sur ce modèle, et à propos du discours politique, on peut lire en français un texte traduit de van Dijk lui-même ainsi qu'une présentation du modèle subtilement commenté par R. Micheli dans Semen 21, 2006.

3. Peut-on voir dans le préconscient l'inscription des prédiscours (concept proposé par M.-A. Paveau 2006, et qu'on peut définir brièvement comme un ensemble de cadres sémantiques cognitifs responsables de la production et de la circulation des discours) ? C'est une question qui reste à discuter...

4. En tapant sur Google « sauter comme un cabri + Europe ", le 22 mai 2006, on a obtenu 555 liens et on a retrouvé l'énoncé originel et son contexte (ainsi que les reprises et reformulations auxquelles il a donné lieu depuis) : «Bien entendu, on peut sauter sur sa chaise comme un cabri en disant l'Europe ! l'Europe ! l'Europe ! mais cela n'aboutit à rien et cela ne signifie rien » (de Gaulle).

5. Mais dans un co(n)texte différent: elle est alors prêtée à Jany le Pen dans des propos qu'elle tient sur son mari : «Il est la force tranquille dont la France a besoin » (voir par exemple Araújo Carreira $2004: 160$ ).

6. Avoir eu l'expérience d'un tremblement de terre fait, dès qu'on sent la terre bouger, remonter en mémoire la désignation qu'on connaît du phénomène ainsi que l'expérience sensorielle qu'on a mémorisée (rôle de la mémoire perceptive ?). Mais des événements de l'histoire, qu'on n'a pas vécus, peuvent s'inscrire en mémoire avec les représentations qu'on a lues, entendues, vues, non seulement dans les livres d'histoire, mais également dans les fictions théâtrales, cinématographiques ou télévisuelles : il y aurait alors un traitement cognitif « multimodal », avec du discours, de l'oral, de l'écrit, de l'iconique, du son... et même de l'émotion.

7. Voir par exemple les ouvrages de Jacques Le Goff, de Pierre Nora ou de Paul Ricœur. Mais les écrivains comme les chercheurs en littérature se réfèrent également aux phénomènes de mémoire et de mémorisation (comme l'attestent les ouvrages récents de Tadié et Tadié ainsi que de Lemardeley et alii éds).

8. Je ne ferai pas d'hypothèses sur les formes de ce traitement cognitif, qui ne relève pas de la compétence du linguiste. Je ne prendrai donc pas position sur la pertinence des modèles symboliques ou des modèles connexionnistes pour expliquer les phénomènes de mémorisation ou de rappel (voir pour une synthèse Tiberghien 1997). Je me contenterai de discuter des formes de mémoire mises au jour par l'analyse discursive.

9. Pour un modèle d'analyse du discours qui articule représentations mentales, représentations discursives et représentations sociales, voir Rakotonoelina 2000.

10. Cité dans Adam 2004, p. 9 : A. Berrendonner : «Schématisation et topographique imaginaire du discours », dans Logique, discours et pensée, Berne, Peter Lang, p. 219-220. 


\section{RÉSUMÉS}

L'analyse du discours française, portant à l'origine sur des discours politiques, s'est construite dans les années 1970-1980 en dehors du paradigme cognitiviste. Les mots cognition ou cognitif semblent cependant avoir été inscrits " en creux ", dès qu'il s'est agi de théoriser le fonctionnement $\mathrm{du}$ discours, en particulier derrière les notions de mémoire et d'oubli et dans leurs liaisons implicites avec les notions de contexte, de pensée et d'expérience humaine : l'existence du préconstruit, de l'interdiscours et de la théorie des deux oublis (Pêcheux), les notions de discours transverses et de mémoire interdiscursive (Courtine et Lecomte), le mot « qui n'oublie jamais son trajet ", et donc les discours qu'il a déjà rencontrés (Bakhtine) ainsi que la notion de mémoire collective (Halbwachs) renvoient sans le dire explicitement à du cognitif. On essaie ici de démêler d'abord ce qu'on entend par mémoire en analyse du discours, depuis Courtine et Lecomte, et dans le cadre actuel d'une sémantique discursive renouvelée par la prise en compte des relations entre le sujet (social) et la réalité (sociale) telle qu'il la perçoit, qu'il l'interprète et qu'il la catégorise avant de la représenter pour d'autres. On s'interroge ensuite, à la lumière des analyses effectuées sur des corpus de la presse quotidienne française, sur ce que les mots et les dires transportent avec eux (fils verticaux du discours) lorsqu'ils s'inscrivent dans la matérialité discursive (fil horizontal du discours).Ce qui permettra de discuter des formes de mémoire (mémoires épisodique, perceptive, procédurale, sémantique et mémoire de travail) qui sont alors convoquées et du rôle de l'allusion dans la mémorisation et le rappel, voire la construction, des savoirs. On montre enfin comment l'appel à la mémoire des mots et des dires intervient dans l'éclairage proposé par l'énonciateur au fil du déroulement du texte, en particulier dans des configurations discursives comme l'explication médiatique ou l'argumentation, c'est-à-dire dans le cadre de la schématisation élaborée par J.-B. Grize : une organisation raisonnée de contenus de pensée visant à modifier les représentations cognitives des destinataires.

French discourse analysis, which originally centered on political discourse, developed during the seventies and eighties outside the cognitive paradigm. The words "cognition" and "cognitive" seem nonetheless to have been reintroduced implicitly as soon as the question arose of theorizing the functioning of discourse, in particular behind the notions of memory and forgetting and in their implicit links with ideas of context, thought and human experience: the existence of the pre-constructed, of inter-discourse and Pêcheux's double forgetting theory, notions of transverse discourse and inter-discourse memory (Courtine and Lecomte), the idea of the word "that never forgets where it has been" and thus the discourses it has already encountered (Bakhtine) as well as the notion of collective memory (Halbwachs) point, although not explicitly, to the cognitive. This article attempts first of all to unravel what is meant by memory in discourse analysis since Courtine and Lecomte and within the present framework of a discursive semantic theory which has been renewed by the taking into account of the relations between the (social) subject and (social) reality as he perceives, interprets and categorizes it before representing it for others. We then examine, in the light of analyses carried out on a corpus from the French daily press, what words and speech bring with them (vertical threads of discourse) when they are inscribed in the materiality of discourse (horizontal thread of discourse). This will allow us to talk about the forms of memory (episodic, perceptive, 
procedural, semantic and working memory) which are evoked and the role of allusion in the memorization and recall, or even in the construction, of knowledge. Finally we show how the appeal to the memory of words and speech can be used by the speaker to modify the perception of a text as it unfurls, in particular in discursive configurations like media explanation of argumentation, in other words within the framework of the model elaborated by J.-B. Grize: a reasoned organization of thought content, the aim of witch is to modify the cognitive representations of those addressed.

INDEX

Mots-clés : allusion, analyse du discours française, dialogisme, interdiscours, intertexte, intratexte, mémoire collective, mémoire interdiscursive, presse quotidienne, représentation Keywords : allusion, collective memory, daily press, dialogism, french discourse analysis, interdiscourse, interdiscursive memory, intertext, intratext

\section{AUTEUR}

\section{SOPHIE MOIRAND}

Cediscor-Syled, Université Sorbonne nouvelle - Paris 3 